\title{
Modeling of Carrier Transport in Nanowires ${ }^{\star}$
}

\author{
T. Gurov, E. Atanassov, M. Nedjalkov, and I. Dimov \\ IPP, Bulgarian Academy of Sciences, Sofia, Bulgaria \\ \{gurov, emanouil\}@parallel.bas.bg, ivdimov@bas.bg \\ Institute for Microelectronics, TU Vienna, Austria \\ mixi@iue.tuwien.ac.at \\ Centre for Advanced Computing and Emerging Technologies \\ School of Systems Engineering, The University of Reading \\ Whiteknights, PO Box 225, Reading, RG6 6AY, UK \\ i.t.dimov@reading.ac.uk
}

\begin{abstract}
We consider a physical model of ultrafast evolution of an initial electron distribution in a quantum wire. The electron evolution is described by a quantum-kinetic equation accounting for the interaction with phonons. A Monte Carlo approach has been developed for solving the equation. The corresponding Monte Carlo algorithm is NP-hard problem concerning the evolution time. To obtain solutions for long evolution times with small stochastic error we combine both variance reduction techniques and distributed computations. Grid technologies are implemented due to the large computational efforts imposed by the quantum character of the model.
\end{abstract}

\section{Introduction}

The Monte Carlo (MC) methods provide approximate solutions by performing statistical sampling experiments. These methods are based on simulation of random variables whose mathematical expectations are equal to a functional of the problem solution.

Many problems in the transport theory and related areas can be described mathematically by a second kind Fredholm integral equation.

$$
f=\mathbb{K}(f)+\phi
$$

In general the physical quantities of interest are determined by functionals of the type:

$$
J(f) \equiv(g, f)=\int_{G} g(x) f(x) d x,
$$

where the domain $G \subset \mathbb{R}^{d}$ and a point $x \in G$ is a point in the Euclidean space $\mathbb{R}^{d}$. The functions $f(x)$ and $g(x)$ belong to a Banach space $X$ and to the adjoint space $X^{*}$, respectively, and $f(x)$ is the solution of (1).

The mathematical concept of the MC approach is based on the iterative expansion of the solution of (1): $f_{s}=\mathbb{K}\left(f_{s-1}\right)+\phi, s=1,2, \ldots$, where $s$ is the number of iterations.

\footnotetext{
* Supported by the Bulgarian Ministry of Education and Science, under grant I-1405/2004.
} 
Thus we define a Neumann series $f_{s}=\phi+\mathbb{K}(\phi)+\ldots+\mathbb{K}^{s-1}(\phi)+\mathbb{K}^{s}\left(f_{0}\right), s>1$, where $\mathbb{K}^{s}$ means the $s$-th iteration of $\mathbb{K}$. In the case when the corresponding infinite series converges then the sum is an element $f$ from the space $X$ which satisfies the equation (11). The Neumann series, replaced in (2), gives rise to a sum of consecutive terms which are evaluated by the MC method with the help of random estimators. A random variable $\xi$ is said to be a MC estimator for the functional (2) if the mathematical expectation of $\xi$ is equal to $J(f): E \xi=J(f)$. Therefore we can define a MC method $\bar{\xi}=\frac{1}{N} \sum_{i=1}^{N} \xi^{(i)} \stackrel{P}{\longrightarrow} J(f)$, where $\xi^{(1)}, \ldots, \xi^{(N)}$ are independent values of $\xi$ and $\stackrel{P}{\longrightarrow}$ means stochastic convergence as $N \longrightarrow \infty$. The rate of convergence is evaluated by the law of the three sigmas: convergence (see [1]): $P\left(|\bar{\xi}-J(f)|<3 \frac{\sqrt{V a r \xi}}{\sqrt{N}}\right) \approx$ 0.997, where $\operatorname{Var}(\xi)=E \xi^{2}-E^{2} \xi$ is the variance. It is seen that, when using a random estimator, the result is obtained with a statistical error [12]. As $N$ increases, the statistical error decreases as $N^{-1 / 2}$.

Thus, there are two types of errors - systematic (a truncation error) and stochastic (a probability error) [2]. The systematic error depends on the number of iterations of the used iterative method, while the stochastic error is related to the the probabilistic nature of the MC method. The MC method still does not determine the computation algorithm: we must specify the modeling function (sampling rule) $\Theta=F\left(\beta_{1}, \beta_{2}, \ldots\right.$, ), where $\beta_{1}, \beta_{2}, \ldots$, are uniformly distributed random numbers in the interval $(0,1)$. Now both relations the MC method and the sampling rule define a $\mathrm{MC}$ algorithm for estimating $J(f)$. The case when $g=\delta\left(x-x_{0}\right)$ is of special interest, because we are interested in calculating the value of $f$ at $x_{0}$, where $x_{0} \in G$ is a fixed point.

Every iterative algorithm uses a finite number of iterations $s$. In practice we define a MC estimator $\xi_{s}$ for computing the functional $J\left(f_{s}\right)$ with a statistical error. On the other hand $\xi_{s}$ is a biased estimator for the functional $J(f)$ with statistical and truncation errors. The number of iterations can be a random variable when an $\varepsilon$-criterion is used to truncate the Neumann series or the corresponding Markov chain in the MC algorithm. The stochastic convergence rate is approximated by $O\left(N^{-1 / 2}\right)$. In order to accelerate the convergence rate of the MC methods several techniques have been developed. Variance reductions techniques, like antithetic varieties, stratification and importance sampling [1], reduce the variance which is a quantity to measure the probabilistic uncertainly. Parallelism is an another way to accelerate the convergence of a MC computation. If $n$ processors execute $n$ independence of the MC computation using nonoverlaping random sequences, the accumulated result has a variance $n$ times smaller than that of a single copy.

\section{Physical Model}

The early time dynamics of highly non-equilibrium semiconductor electrons is rich of quantum phenomena which are basic components of the physics of the modern micro- and nano-electronics [4]. Confined systems are characterized by small spatial scales where another classical assumption - this for electron-phonon scattering occuring at a well defined position - loses its validity. These scales become relevant for the process of evolution of an initial electron distribution, locally excited or injected into a 
semiconductor nanowire. Beyond-Boltzmann transport models for wire electrons have been recently derived [3] in terms of the Wigner function. They appear as inhomogeneous conterparts of Levinson's and the Barker-Ferry's equations. The later is a physically more refined model, which accounts for the finite lifetime of the electrons due to the interaction with the phonons.

The corresponding physical process can be summarized as follows. An initial nonequilibrium electron distribution is created locally in a nanowire by e.g. an optical excitation. The electrons begin to propagate along the wire, where they are characterized by two variables: the position $z$ and the component of the wave vector is $k_{z}$. We note that these are Wigner coordinates so that a classical interpretation as "position and momentum of the electron" is not relevant. A general, time-dependent electric field $E(t)$ can be applied along the nanowire. The field changes the wave vector with the time: $k_{z}\left(t^{\prime}\right)=k_{z}-\int_{t^{\prime}}^{t} e E(\tau) / \hbar d \tau$ where $k_{z}$ is the wave vector value at the initialization time $t$. Another source of modifications of the wave vector are dissipative processes of electron-phonon interaction, responsible for the energy relaxation of the electrons.

The following considerations simplify the problem thus allowing to focus on the numerical aspects: (i) We regard the Levinson type of equation which bears the basic numerical properties of the physical model:

$$
\begin{aligned}
& \left(\frac{\partial}{\partial t}+\frac{\hbar k_{z}}{m} \nabla_{z}\right) f_{w}\left(z, k_{z}, t\right)=\int d \mathbf{k}^{\prime} \int_{0}^{t} d t^{\prime} \\
& \left\{S\left(k_{z}, k_{z}^{\prime}, \mathbf{q}_{\perp}^{\prime}, t, t^{\prime}\right) f_{w}\left(z\left(t^{\prime}\right), k_{z}{ }^{\prime}\left(t^{\prime}\right), t^{\prime}\right)-S\left(k_{z}^{\prime}, k_{z}, \mathbf{q}_{\perp}^{\prime}, t, t^{\prime}\right) f_{w}\left(z\left(t^{\prime}\right), k_{z}\left(t^{\prime}\right), t^{\prime}\right)\right\}
\end{aligned}
$$

where $\int_{G} d^{3} \mathbf{k}^{\prime}=\int d \mathbf{q}_{\perp}^{\prime} \int_{-Q_{2}}^{Q_{2}} d k_{z}^{\prime}$ and the domain $G$ is specified in the next section. The spatial part $z\left(t^{\prime}\right)$ of the trajectory is initialized by the value $z$ at time $t: z\left(t^{\prime}\right)=$ $z-\frac{\hbar}{m} \int_{t^{\prime}}^{t}\left(k_{z}(\tau)-\frac{q_{z}^{\prime}}{2}\right) d \tau ; q_{z}^{\prime}=k_{z}-k_{z}^{\prime}$. The scattering function $S$ is

$$
\begin{aligned}
& S\left(k_{z}, k_{z}^{\prime}, \mathbf{q}_{\perp}^{\prime}, t, t^{\prime}\right)=\frac{2 V}{(2 \pi)^{3}}\left|\Gamma\left(\mathbf{q}_{\perp}^{\prime}\right) \mathcal{F}\left(\mathbf{q}_{\perp}^{\prime}, k_{z}-k_{z}^{\prime}\right)\right|^{2} \\
& \times\left[\left(n\left(\mathbf{q}^{\prime}\right)+\frac{1}{2} \pm \frac{1}{2}\right) \cos \left(\int_{t^{\prime}}^{t} \frac{\left(\epsilon\left(k_{z}(\tau)\right)-\epsilon\left(k_{z}^{\prime}(\tau)\right) \pm \hbar \omega_{\mathbf{q}^{\prime}}\right) d \tau}{\hbar}\right)\right] .
\end{aligned}
$$

The electron-phonon coupling $\mathcal{F}$ is chosen for the case of Fröhlich polar optical interaction:

$$
\begin{aligned}
\mathcal{F} & \left(\mathbf{q}_{\perp}^{\prime}, k_{z}-k_{z}^{\prime}\right) \\
& =-\left[\frac{2 \pi e^{2} \omega_{\mathbf{q}^{\prime}}}{\hbar V}\left(\frac{1}{\varepsilon} \infty-\frac{1}{\varepsilon_{s}}\right) \frac{1}{\left(\mathbf{q}^{\prime}\right)^{2}}\right]^{\frac{1}{2}} ; \Gamma\left(\mathbf{q}_{\perp}^{\prime}\right)=\int d \mathbf{r}_{\perp}\left|\Psi\left(\mathbf{r}_{\perp}\right)\right|^{2} e^{i \mathbf{q}_{\perp}^{\prime} \cdot \mathbf{r}_{\perp},}
\end{aligned}
$$

where $\left(\varepsilon_{\infty}\right)$ and $\left(\varepsilon_{s}\right)$ are the optical and static dielectric constants. $\Gamma\left(\mathbf{q}_{\perp}^{\prime}\right)$ is the Fourier transform of the square of the ground state wave function. Thus we come to the second simplifying assumption: (ii) Very low temperatures are assumed, so that the electrons reside in the ground state $\Psi\left(\mathbf{r}_{\perp}\right)$ in the plane normal to the wire. The phonon distribution is described by the Bose function, $n\left(\mathbf{q}^{\prime}\right)=\left(e^{\frac{\hbar \omega_{\mathbf{q}^{\prime}}}{\kappa T}}-1\right)^{-1}$ with $K$ the Boltzmann 
constant and $T$ is the temperature of the crystal. $\hbar \omega_{\mathbf{q}^{\prime}}$ is the phonon energy which generally depends on $\mathbf{q}^{\prime}=\left(\mathbf{q}_{\perp}^{\prime}, q_{z}^{\prime}\right)$, and $\varepsilon\left(k_{z}\right)=\left(\hbar^{2} k_{z}^{2}\right) / 2 m$ is the electron energy. (iii) Finally we consider the case of no electric field applied along the wire. Next we need to transform the transport equation into the corresponding integral form (1):

$$
\begin{aligned}
& f_{w}\left(z, k_{z}, t\right)=f_{w 0}\left(z-\frac{\hbar k_{z}}{m} t, k_{z}\right) \\
+ & \int_{0}^{t} d t^{\prime \prime} \int_{t^{\prime \prime}}^{t} d t^{\prime} \int_{G} d^{3} \mathbf{k}^{\prime}\left\{K_{1}\left(k_{z}, k_{z}^{\prime}, \mathbf{q}_{\perp}^{\prime}, t^{\prime}, t^{\prime \prime}\right) f_{w}\left(z+z_{0}\left(k_{z}, q_{z}^{\prime}, t, t^{\prime}, t^{\prime \prime}\right), k_{z}^{\prime}, t^{\prime \prime}\right)\right\} \\
+ & \int_{0}^{t} d t^{\prime \prime} \int_{t^{\prime \prime}}^{t} d t^{\prime} \int_{G} d^{3} \mathbf{k}^{\prime}\left\{K_{2}\left(k_{z}, k_{z}^{\prime}, \mathbf{q}_{\perp}^{\prime}, t^{\prime}, t^{\prime \prime}\right) f_{w}\left(z+z_{0}\left(k_{z}, q_{z}^{\prime}, t, t^{\prime}, t^{\prime \prime}\right), k_{z}, t^{\prime \prime}\right)\right\},
\end{aligned}
$$

where $z_{0}\left(k_{z}, q_{z}^{\prime}, t, t^{\prime}, t^{\prime \prime}\right)=-\frac{\hbar k_{z}}{m}\left(t-t^{\prime \prime}\right)+\frac{\hbar q_{z}^{\prime}}{2 m}\left(t^{\prime}-t^{\prime \prime}\right)$,

$$
K_{1}\left(k_{z}, k_{z}^{\prime}, \mathbf{q}_{\perp}^{\prime}, t^{\prime}, t^{\prime \prime}\right)=S\left(k_{z}^{\prime}, k_{z}, \mathbf{q}_{\perp}^{\prime}, t^{\prime}, t^{\prime \prime}\right)=-K_{2}\left(k_{z}^{\prime} . k_{z}, \mathbf{q}_{\perp}^{\prime}, t^{\prime}, t^{\prime \prime}\right) .
$$

We note that the evolution problem becomes inhomogeneous due to the spatial dependence of the initial condition $f_{w 0}$. The shape of the wire modifies the electron-phonon coupling via the ground state in the normal plane. If the wire cross section is chosen to be a square with side $a$, the corresponding factor $\Gamma$ becomes: $\left|\Gamma\left(q_{x}^{\prime}\right) \Gamma\left(q_{y}^{\prime}\right)\right|^{2}=$ $\left|\Gamma\left(\mathbf{q}_{\perp}^{\prime}\right)\right|^{2}=\left(\frac{4 \pi^{2}}{q_{x}^{\prime} a\left(\left(q_{x}^{\prime} a\right)^{2}-4 \pi^{2}\right)}\right)^{2} 4 \sin ^{2}\left(a q_{x}^{\prime} / 2\right)\left(\frac{4 \pi^{2}}{q_{y}^{\prime} a\left(\left(q_{y}^{\prime} a\right)^{2}-4 \pi^{2}\right)}\right)^{2} 4 \sin ^{2}\left(a q_{y}^{\prime} / 2\right)$. We note that the Neumann series of such type integral equations as (4) converges [5] and it can be evaluated by a MC estimator [2].

\section{The Monte Carlo Method}

The values of the physical quantities are expressed by the following general functional of the solution of (4):

$$
J_{g}(f) \equiv(g, f)=\int_{0}^{\mathcal{T}} \int_{D} g\left(z, k_{z}, t\right) f_{w}\left(z, k_{z}, t\right) d z d k_{z} d t
$$

by a MC method. Here we specify that the phase space point $\left(z, k_{z}\right)$ belongs to a rectangular domain $D=\left(-Q_{1}, Q_{1}\right) \times\left(-Q_{2}, Q_{2}\right)$, and $t \in(0, \mathcal{T})$. The function $g\left(z, k_{z}, t\right)$ depends on the quantity of interest. In particular the Wigner function, the wave vector and density distributions, and the energy density are given by:

$$
\begin{aligned}
& \text { (i) } g_{W}\left(z, k_{z}, t\right)=\delta\left(z-z_{0}\right) \delta\left(k_{z}-k_{z, 0}\right) \delta\left(t-t_{0}\right), \\
& \text { (ii) } g_{n}\left(z, k_{z}, t\right)=\delta\left(k_{z}-k_{z, 0}\right) \delta\left(t-t_{0}\right), \\
& \text { (iii) } g_{k}\left(z, k_{z}, t\right)=\delta\left(z-z_{0}\right) \delta\left(t-t_{0}\right), \\
& \text { (iv) } g\left(z, k_{z}, t\right)=\epsilon\left(k_{z}\right) \delta\left(z-z_{0}\right) \delta\left(t-t_{0}\right) / g_{n}\left(z, k_{z}, t\right),
\end{aligned}
$$

We construct a biased MC estimator for evaluating the functional (5) using backward time evolution of the numerical trajectories in the following way: 


$$
\xi_{s}\left[J_{g}(f)\right]=\frac{g\left(z, k_{z}, t\right)}{p_{i n}\left(z, k_{z}, t\right)} W_{0} f_{w, 0}\left(., k_{z}, 0\right)+\frac{g\left(z, k_{z}, t\right)}{p_{i n}\left(z, k_{z}, t\right)} \sum_{j=1}^{s} W_{j}^{\alpha} f_{w, 0}\left(., k_{z, j}^{\alpha}, t_{j}\right)
$$

where

$$
\begin{aligned}
& f_{w, 0}\left(., k_{z, j}^{\alpha}, t_{j}\right) \\
& = \begin{cases}f_{w, 0}\left(z+z_{0}\left(k_{z, j-1}, k_{z, j-1}-k_{z, j}, t_{j-1}, t_{j}^{\prime}, t_{j}\right), k_{z, j}, t_{j}\right), & \text { if } \alpha=1, \\
f_{w, 0}\left(z+z_{0}\left(k_{z, j-1}, k_{z, j}-k_{z, j-1}, t_{j-1}, t_{j}^{\prime}, t_{j}\right), k_{z, j-1}, t_{j}\right), & \text { if } \alpha=2,\end{cases} \\
& W_{j}^{\alpha}=W_{j-1}^{\alpha} \frac{K_{\alpha}\left(k_{z j-1}, \mathbf{k}_{j}, t_{j}^{\prime}, t_{j}\right)}{p_{\alpha} p_{t r}\left(\mathbf{k}_{j-1}, \mathbf{k}_{j}, t_{j}^{\prime}, t_{j}\right)}, W_{0}^{\alpha}=W_{0}=1, \quad \alpha=1,2, j=1, \ldots, s .
\end{aligned}
$$

The probabilities $p_{\alpha},(\alpha=1,2)$ are chosen to be proportional to the absolute value of the kernels in (4). $p_{i n}\left(z, k_{z}, t\right)$ and $p_{t r}\left(\mathbf{k}, \mathbf{k}^{\prime}, t^{\prime}, t^{\prime \prime}\right)$, which are an initial density and a transition density, are chosen to be tolerant 1 to the function $g\left(z, k_{z}, t\right)$ and the kernels, respectively. The first point $\left(z, k_{z_{0}}, t_{0}\right)$ in the Markov chain is chosen using the initial density, where $k_{z 0}$ is the third coordinate of the wave vector $\mathbf{k}_{0}$. Next points $\left(k_{z j}, t_{j}^{\prime}, t_{j}\right) \in\left(-Q_{2}, Q_{2}\right) \times\left(t_{j}, t_{j-1}\right) \times\left(0, t_{j-1}\right)$ of the Markov chain:

$$
\left(k_{z 0}, t_{0}\right) \rightarrow\left(k_{z 1}, t_{1}^{\prime}, t_{1}\right) \rightarrow \ldots \rightarrow\left(k_{z j}, t_{j}^{\prime}, t_{j}\right) \rightarrow \ldots \rightarrow\left(k_{z_{s}}, t_{s}^{\prime}, t_{s}\right), j=1,2, \ldots, s
$$

do not depend on the position $z$ of the electrons. They are sampled using the transition density $p_{t r}\left(\mathbf{k}, \mathbf{k}^{\prime}, t^{\prime}, t^{\prime \prime}\right)$. The $z$ coordinate of the generated wave vectors is taken for the Markov chain, while the normal coordinates give values for $\mathbf{q}_{\perp}$. As the integral on $\mathbf{q}_{\perp}$ can be assigned to the kernel these values do not form a Markov chain but are independent on the consecutive steps. The time $t_{j}^{\prime}$ conditionally depends on the selected time $t_{j}$. The Markov chain terminates in time $t_{s}<\varepsilon_{1}$, where $\varepsilon_{1}$ is related to the truncation error introduced in first section. Now the functional (5) can be evaluated by $N$ independent samples of the obtained estimator, namely, $\frac{1}{N} \sum_{i=1}^{N}\left(\xi_{s}\left[J_{g}(f)\right]\right)_{i} \stackrel{P}{\longrightarrow} J_{g}\left(f_{s}\right) \approx J_{g}(f)$, where $\stackrel{P}{\longrightarrow}$ means stochastic convergence as $N \rightarrow \infty ; f_{s}$ is the iterative solution obtained by the Neumann series of (4), and $s$ is the number of iterations. To define a MC algorithm we have to specify the initial and transition densities, as well the modeling function (or sampling rule). The modeling function describes the rule needed to calculate the states of the Markov chain by using uniformly distributed random numbers in the interval $(0,1)$. The transition density is chosen: $p_{t r}\left(\mathbf{k}, \mathbf{k}^{\prime}, t^{\prime}, t^{\prime \prime}\right)=p\left(\mathbf{k}^{\prime} / \mathbf{k}\right) p\left(t, t^{\prime}, t^{\prime \prime}\right)$, where $\left.p\left(t, t^{\prime}, t^{\prime \prime}\right)=p\left(t, t^{\prime \prime}\right) p\left(t^{\prime} / t^{\prime \prime}\right)=\frac{1}{t} \frac{1}{\left(t-t^{\prime \prime}\right)} p\left(\mathbf{k}^{\prime} / \mathbf{k}\right)=c_{1} /\left(\mathbf{k}^{\prime}-\mathbf{k}\right)^{2}\right]$, and $c_{1}$ is the normalized constant. Thus, by simulating the Markov chain under consideration, the desired physical quantities (values of the Wigner function, the wave vector relaxation, the electron and energy density) can be evaluated simultaneously.

\section{Grid Implementation and Numerical Results}

The computational complexity of an MC algorithm can be measured by the quantity $C C=N \times \tau \times M\left(s_{\varepsilon_{1}}\right)$. The number of the random walks, $N$, and the average number

\footnotetext{
${ }^{1} r(x)$ is tolerant of $g(x)$ if $r(x)>0$ when $g(x) \neq 0$ and $r(x) \geq 0$ when $g(x)=0$.
} 


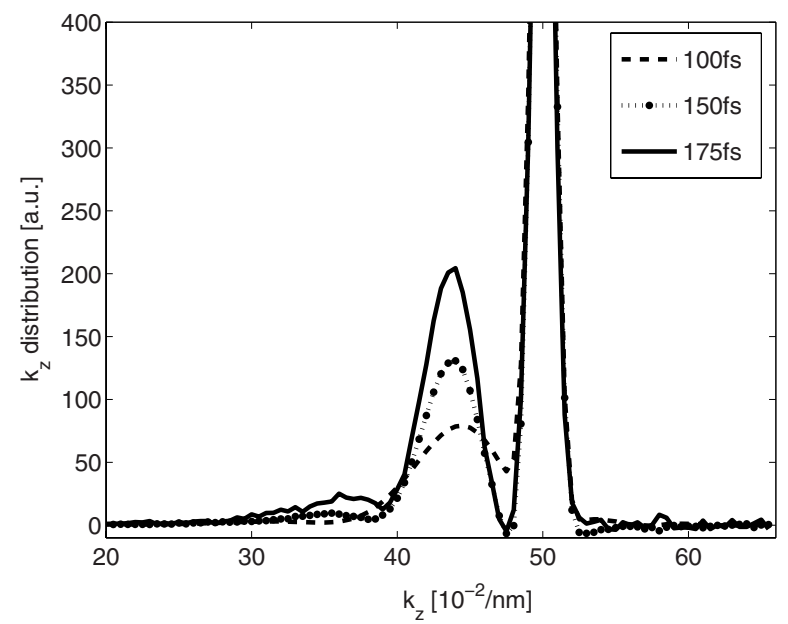

Fig. 1. Wave vector relaxation of the highly non-equilibrium initial condition. The quantum solution shows broadening of the replicas. Electrons appear in the classically forbidden region above the initial condition.

of transitions in the Markov chain, $M\left(s_{\varepsilon_{1}}\right)$, are related to the stochastic and systematic errors [5]. The mean time for modeling one transition, $\tau$, depends on the complexity of the transition density functions and on the sampling rule, as well as on the choice of the random number generator (rng). It is proved [5]6] that the stochastic error has order $O\left(\exp \left(c_{2} t\right) / N^{1 / 2}\right)$, where $t$ is the evolution time and $c_{2}$ is a constant depending on the kernels of the quantum kinetic equation under consideration. This estimate shows that when $t$ is fixed and $N \rightarrow \infty$ the error decreases, but for large $t$ the factor $\exp \left(c_{2} t\right)$ looks ominous. Therefore, the algorithm solves an $N P$-hard problem concerning the evolution time. To solve this problem for long evolution times with small stochastic error we have to combine both MC variance reduction techniques and distributed or parallel computations. By using the Grid environment provided by the EGEE-II project middleware [ [8] we were able to reduce the computing time of the $\mathrm{MC}$ algorithm under consideration. The simulations are parallelized on the existing Grid infrastructure by splitting the underlying random number sequences. The numerical results discussed in Fig. 1 are obtained for zero temperature and GaAs material parameters: the electron effective mass is 0.063 , the optimal phonon energy is $36 \mathrm{meV}$, the static and optical dielectric constants are $\varepsilon_{s}=10.92$ and $\varepsilon_{\infty}=12.9$. The initial condition is a product of two Gaussian distributions of the energy and space. The $k_{z}$ distribution corresponds to a generating laser pulse with an excess energy of about $150 \mathrm{meV}$.This distribution was estimated for 130 points in the interval $(0,66)$, where $Q_{2}=66 \times 10^{7} \mathrm{~m}^{-1}$. The $z$ distribution is centered around zero (see Figures 2-3) and it is estimated for 400 points in the interval $\left(-Q_{1}, Q_{1}\right)$, where $Q_{1}=400 \times 10^{9} \mathrm{~m}^{-1}$. The side $a$ of the wire is chosen to be 10 nanometers. The SPRNG library has been used to produce independent and

\footnotetext{
${ }^{2}$ The Enabling Grids for E-sciencE-II (EGEE-II) project is funded by the EC under grand INFSO-RI-031688. For more information see http://www.eu-egee.org/.
} 


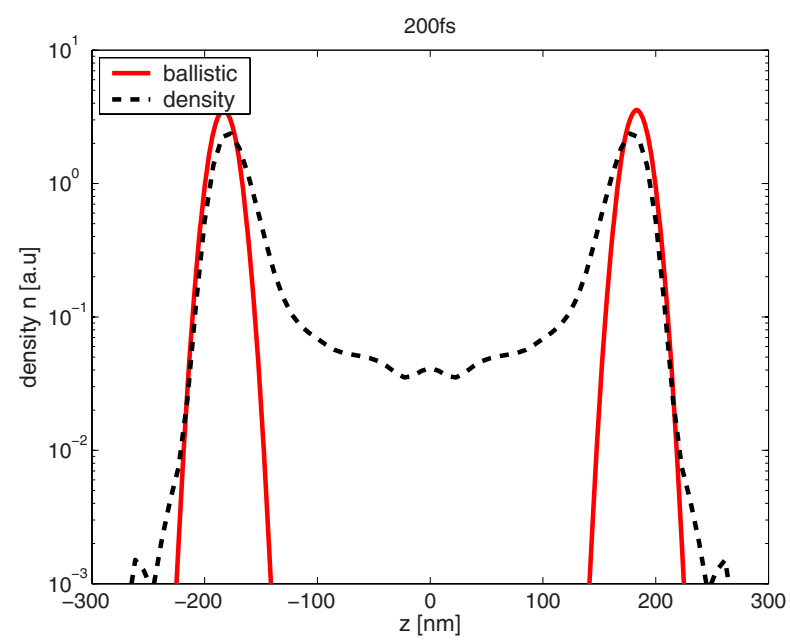

Fig. 2. Electron density along the wire after $200 \mathrm{fs}$. The ballistic curve outlines the largest distance which can be reached by classical electrons. The quantum solution reaches larger distances due to the electrons scattered in the classically forbidden energy region.

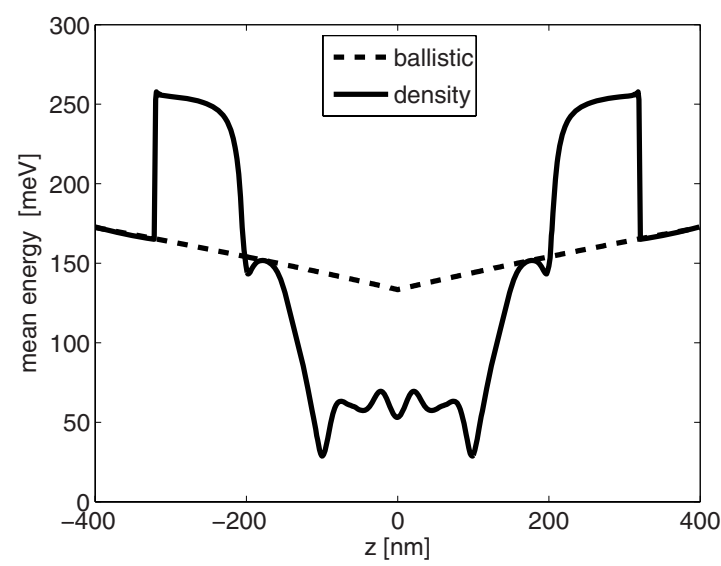

Fig. 3. Energy density after 175 fs evolution time. A comparison with the ballistic curve shows that the mean kinetic energy per particle is lower in the central region. On contrary hot electrons reside in the regions apart from the center. These electrons are faster than the ballistic ones and thus cover larger distances during the evolution.

non-overlapping random sequences [9]. Successful tests of the algorithm were performed at the Bulgarian SEE-GRID 3 sites. The MPI implementation was MPICH 1.2.6, and the execution is controlled from the Computing Element via the Torque batch

\footnotetext{
${ }^{3}$ South Eastern European GRid-enabled eInfrastructure Development-2 (SEE-GRID-2) project is funded by the EC under grand FP6-RI-031775. For more information see http://www.seegrid.eu/.
} 
Table 1. The CPU time (seconds) for all points (in which the physical quantities are estimated), the speed-up, and the parallel efficiency. The number of random walks is $N=100000$. The evolution time is $100 \mathrm{fs}$.

\begin{tabular}{cccc}
\hline \hline Number of CPUs & CPU Time (s) Speed-up & Parallel Efficiency \\
\hline \hline 2 & 9790 & - & - \\
4 & 4896 & 1.9996 & 0.9998 \\
6 & 3265 & 2.9985 & 0.9995 \\
\hline \hline
\end{tabular}

system. The timing results for evolution time $\mathrm{t}=100$ femtoseconds are shown in Table 1 . The parallel efficiency is close to $100 \%$.

\section{Conclusion}

A quantum-kinetic model for the evolution of an initial electron distribution in a quantum wire has been introduced in terms of the electron Wigner function. The physical quantities, expressed as functionals of the Wigner function are evaluated within a stochastic approach. The developed MC method is characterized by the typical for quantum algorithms computational demands. The stochastic variance grows exponentially with the evolution time. The importance sampling technique is used to reduce the variance of the MC method. The suggested MC algorithm evaluates simultaneously the desired physical quantities using simulation of Markov chain under consideration. Grid technologies are implemented to reduce computational efforts.

\section{References}

1. M.A. Kalos, P.A. Whitlock, Monte Carlo methods, Wiley Interscience, New York (1986).

2. G.A. Mikhailov, New MC Methods with Estimating Derivatives, Utrecht, The Netherlands (1995).

3. M. Nedjalkov et al., "Wigner transport models of the electron-phonon kinetics in quantum wires", Physical Review B, vol. 74, pp. 035311-1-035311-18, (2006).

4. T.C. Schmidt and K. Moehring, "Stochastic Path-Integral Simulation of Quantum Scattering", Physical Review A, vol. 48, no. 5, pp. R3418-R3420, (1993).

5. T.V. Gurov, P.A. Whitlock, "An Efficient Backward Monte Carlo Estimator for Solving of a Quantum Kinetic Equation with Memory Kernel”, Mathematics and Computers in Simulation (60), pp. 85-105, (2002).

6. T.V. Gurov et al., "Femtosecond Relaxation of Hot Electrons by Phonon Emission in Presence of Electric Field", Physica B (314), pp. 301-304, (2002).

7. T.V. Gurov, I.T. Dimov, "A Parallel Monte Carlo Method For Electron Quantum Kinetic Equation", Lect. Notes in Comp. Sci. (2907), Springer-Verlang, pp.151-161, (2004).

8. EGEE Grid Middleware, http://lcg.web.cern.ch/LCG/Sites/releases.html.

9. Scalable Parallel Random Number Generators Library for Parallel Monte Carlo Computations, SPRNG 1.0 and SPRNG 2.0 - http://sprng.cs.fsu.edu . 